Supporting Information for:

\title{
Symmetry-Dependent Optical Properties of Stellated
}

\section{Nanocrystals}

Alison F. Smith, ${ }^{\dagger+}$ Rebecca G. Weiner, ${ }^{\dagger}$ and Sara E. Skrabalak ${ }^{* \dagger}$

${ }^{\dagger}$ Department of Chemistry, Indiana University, Bloomington, Indiana 47405, USA.

NAVSEA Crane, Crane, IN 47522, USA. 


\section{Absorption Spectrum of Pt Y-Tripod}

Figure S1 depicts the FDTD calculated absorption spectrum of a Pt Y-tripod particle with a LSPR of $1356 \mathrm{~nm}$ compared to the experimentally determined value of $1380 \mathrm{~nm}$ by Maksimuk et al. ${ }^{1}$

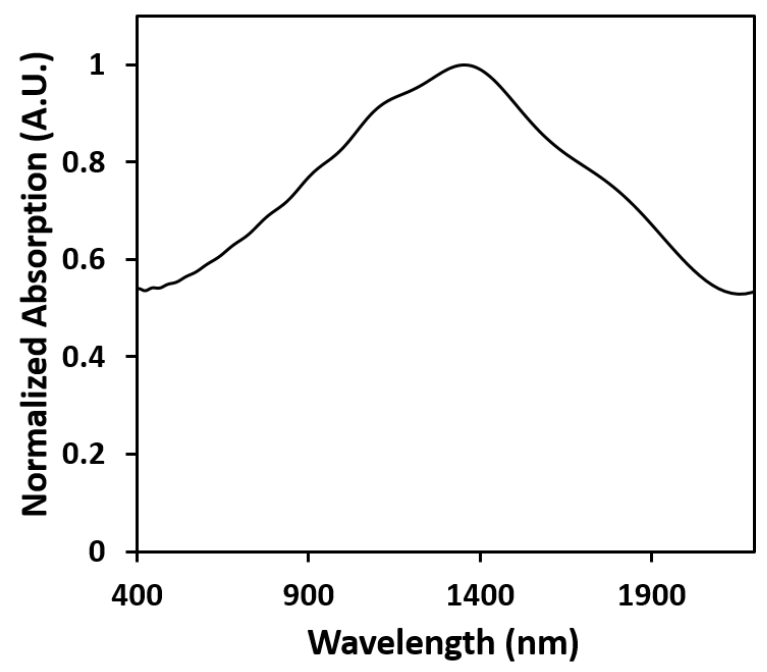

Figure S1. FDTD-calculated absorption spectrum of Pt Y-tripod with arm length of $75 \mathrm{~nm}$. All branch lengths are $75 \mathrm{~nm}$.

\section{Electric Field Vector Map}

Figure S2 a,b depicts the electric field vectors traveling along the 4 branches for the $\mathrm{k}-\mathrm{C}_{2}$ case. Here, the total charge will be accumulated on 4 tips; however the overall dipole is equivalent to that of the $\mathrm{k}-\mathrm{C}_{4}$ case in which the total charge is accumulated on only 2 tips.
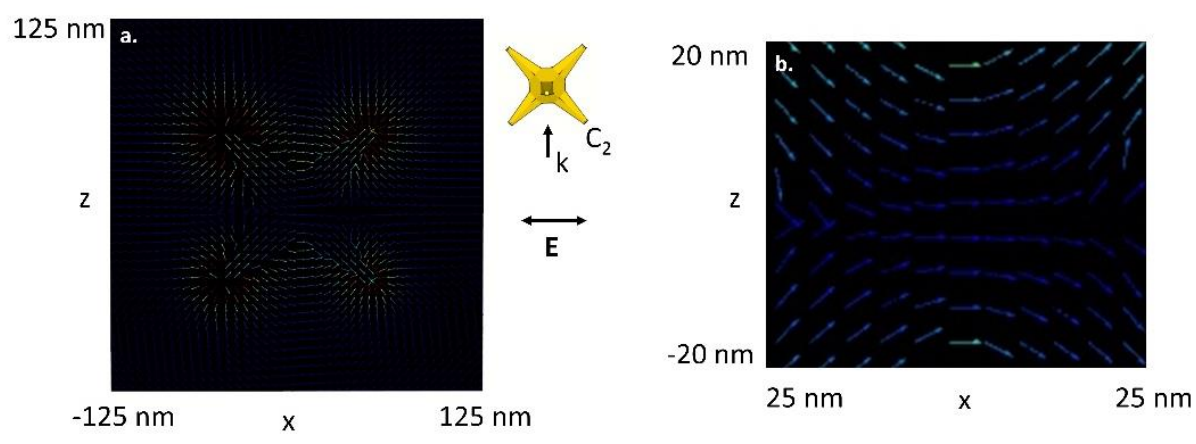

Figure S2. a) Electric field vector map for the Au hexapod with $\mathrm{k}$ along the $\mathrm{C}_{2}$ axis and $\mathrm{E}$ polarized as shown. For this orientation, the electric field directions along the branches are equivalent to that of a linear charge separation. b) an enlarged image of the electric field lines along branches entering/exiting the core. 


\section{References}

1. Maksimuk, S.; Teng, X.; Yang, H., Roles of Twin Defects in the Formation of Platinum Multipod Nanocrystals. J. Phys. Chem. C 2007, 111, 14312-14319. 